Check for updates

Cite this: Chem. Sci., 2018, 9, 4696

\title{
Self-reporting and refoldable profluorescent single-chain nanoparticles $\uparrow$
}

\author{
Tobias S. Fischer, ${ }^{\text {ab }}$ Sebastian Spann, ${ }^{\text {cd }}$ Qi An, ${ }^{\text {e }}$ Burkhard Luy, ${ }^{\text {cd }}$ Manuel Tsotsalas, ${ }^{\text {ce }}$ \\ James P. Blinco, (D)*af Hatice Mutlu (D)*ab and Christopher Barner-Kowollik (D)*abf
}

We pioneer the formation of self-reporting and refoldable profluorescent single-chain nanoparticles (SCNPs) via the light-induced reaction $\left(\lambda_{\max }=320 \mathrm{~nm}\right)$ of nitroxide radicals with a photo-active crosslinker. Whereas the tethered nitroxide moiety in these polymers fully quenches the luminescence (i.e. fluorescence) of the aromatic backbone, nitroxide trapping of a transient $\mathrm{C}$-radical leads to the corresponding closed shell alkoxyamine thereby restoring luminescence of the folded SCNP. Hence, the polymer in the folded state is capable of emitting light, while in the non-folded state the luminescence is silenced. Under oxidative conditions the initially folded SCNPs unfold, resulting in luminescence switchoff and the reestablishment of the initial precursor polymer. Critically, we show that the luminescence can be repeatedly silenced and reactivated. Importantly, the self-reporting character of the SCNPs was followed by size-exclusion chromatography (SEC), dynamic light scattering (DLS), fluorescence, electron paramagnetic resonance (EPR), nuclear magnetic resonance (NMR) and diffusion ordered NMR spectroscopy (DOSY).

Received 2nd March 2018

Accepted 29th April 2018

DOI: $10.1039 / \mathrm{c} 8 \mathrm{sc0} 01009 \mathrm{a}$

rsc.li/chemical-science are accessible by two pathways: (i) via so-called selective point folding, where the folding motifs are attached at pre-selected points along the polymer chain, which leads to well-defined SCNPS or (ii) via the repeating unit approach, leading to less well-defined SCNP structures, due to the random attachment of the folding units along the polymer chain. ${ }^{8}$ While the collapse of the parent single polymer chain can be induced either by non-covalent ${ }^{9-15}$ or dynamic covalent ${ }^{\mathbf{1 6 - 2 0}}$ interactions, a vast majority of the cross-linking strategies developed so far falls in the category of covalent chemistry. ${ }^{6,21,22}$ For instance, attempting to design SCNPs via radical-coupling chemistry, the groups of Suh, Thayumanavan and Mecerreyes reported the collapse of various polymer functionalities via radical-mediated intramolecular covalent cross-linking. ${ }^{23-25}$ Specifically, azo-bis(isobutyronitrile) (AIBN) was selected as radical initiator for the intramolecular crosslinking of these polymers. On the other hand, Berda and colleagues recently introduced atom transfer radical coupling (ATRC) ${ }^{\mathbf{2}}$ to single-chain folding technology. Intriguingly, SCNP folding by light-induced processes are relatively new to single-chain technology, with only a few reported examples. ${ }^{27-33}$ Critically, to the best of our knowledge, there exists no example of SCNPs that exploit the combination of light-induced chemistry and persistent radicals (such as nitroxides) to induce single-chain folded structures. In fact, the nitroxide radicals are known as one of the rare classes of radicals capable of being handled under ambient conditions on the laboratory bench and find applications in diverse fields as cathodes for light-weight organic batteries, ${ }^{34-36}$ antioxidants, ${ }^{37-39}$ and catalysts ${ }^{40}$ among others. ${ }^{41-44}$ Furthermore, the nitroxide $\dagger$ Electronic supplementary information (ESI) available. See DOI: 10.1039/c8sc01009a 
radicals are also the most exploited paramagnetic sensors. Indeed, they form the basis of spin probes and spin labels, ${ }^{45}$ which are well known in the fields of chemical and biological materials science. Hence, we have also recently reported the synthesis of functional self-reporting switch-on systems, where the fluorescence of a fluorophore is silenced by the unpaired spins of nitroxides and restored by the annihilation of the paramagnetic moiety by reduction to yield a paramagnetic hydroxylamine or by trapping of the radical. ${ }^{46-48}$

Generally, polymers bearing an aromatic backbone are known for their fluorescence properties. Polystyrene (PS) is one less known polymer that in solution and in PS particles displays fluorescence due to monomer fluorescence and $\pi$-stacking, ${ }^{49,50}$ although the aromatic rings in PS are smaller compared to pyrene, for example. Hence, in the current contribution, we exploit the concept of nitroxide containing polymers and their quenching of fluorescence (i.e. luminescence) in PS systems for the preparation of profluorescent single-chain nanoparticles and induce the radical coupling reaction employing a UV responsive bifunctional crosslinker. Unlike noncovalent and dynamic cross-linking chemistry, typical covalent cross-linking delivers static nanoparticles that remain stable and unchanged in response to external stimuli. Importantly, the herein established folding process is fully reversible and self-reporting via luminescence, i.e. the folded state does luminesce, while the luminescence of the exited states in the folded polymers is quenched in the open chain. The thus introduced self-reporting SCNPs that emit information about their folding state constitute a critical step forward as reversibly addressable imaging agents.

\section{Results and discussion}

As polymer scaffold, we prepared well-defined random copolymers based on styrene and 4-(chloromethyl)styrene (CMS) with various molar concentrations of the CMS monomer in the copolymer (5-30 mol\%; see Scheme 1 and Table 1).

To assess the efficiency of the reaction and to determine the optimum conditions, not only the different molar ratios of CMS, but the chain-length of the copolymer was varied (refer to ESI Table S.1†). The copolymers were prepared by a well-established route in our group, using nitroxide mediated polymerization $(\mathrm{NMP})^{27,39,51}$ to afford the precursor polymers poly(styrene-stat-4(chloromethyl)styrene) P1-P4. Subsequently, the copolymers were decorated with 4-carboxy-2,2,6,6-tetramethylpiperidine-1oxyl (4-carboxy-TEMPO) in a nucleophilic substitution reaction to yield the parent copolymers $\mathbf{P 1} \mathbf{1}^{\prime}-\mathbf{P} \mathbf{4}^{\prime}$ for single-chain folding. Although the information obtained is less comprehensive, we adopted ${ }^{1} \mathrm{H}$ NMR analysis for the nitroxide containing polymers to follow the post-polymerization modification reaction, which results in a characteristic downfield shift of the methylene proton resonance of the CMS group between 4.6 to $5.2 \mathrm{ppm}$ (refer to Fig. S3-S6†). Confirmation of the modification was also achieved via SEC measurements (Fig. S13-S15 $\dagger$ ), indicating a shift to higher molecular weights after modification.

Table 1 Molecular characteristics of the parent polymer $\mathrm{P}^{\prime}$ and the resulting SCNPs P8, P9 and P11 as well as the unfolded polymer P10, the molecular weights, dispersities, DLS and DOSY derived hydrodynamic diameter and incorporated mol\% of TEMPO

\begin{tabular}{lllllll}
\hline ID & $M_{\mathrm{n}}{ }^{a} / \mathrm{g} \mathrm{mol}^{-1}$ & $D^{a}$ & $D_{\mathrm{h}, \mathrm{DLS}}{ }^{b}$ & $D_{\mathrm{h}, \mathrm{DOSY}}{ }^{c}$ & Density of $\mathrm{TEMPO}^{d}$ & 1 (eq. $)^{e}$ \\
\hline P4 $^{\prime}$ & 33300 & 1.3 & 3.0 & 3.4 & 3.89 & - \\
P9 & 18500 & 1.5 & 2.8 & 2.6 & 0.43 & 2 \\
P10 & 33400 & 1.4 & 3.2 & 3.5 & 2.16 & - \\
P11 & 16200 & 1.6 & 2.3 & 2.9 & 0.03 & 2
\end{tabular}

${ }^{a}$ Determined via SEC in THF $\left(35^{\circ} \mathrm{C}, 1 \mathrm{~mL} \mathrm{~min}^{-1}\right)$ as eluent, calibrated with PS standards. ${ }^{b}$ Determined via DLS in DMAc $+\operatorname{LiBr}(0.3 \%)$ as solvent. ${ }^{c}$ Determined via DOSY. ${ }^{d}$ Determined by EPR. ${ }^{e}$ Equivalents were calculated to the corresponding amount of TEMPO.

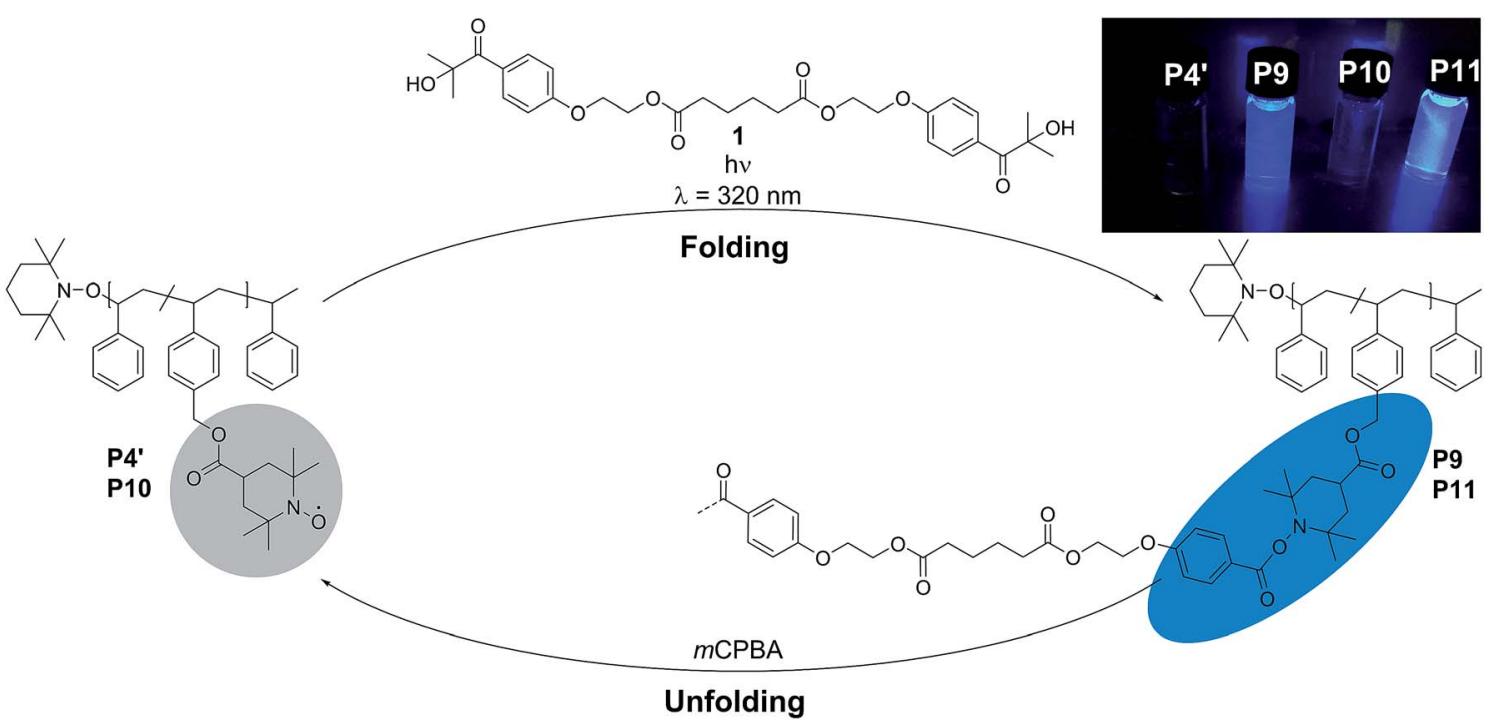

Scheme 1 Folding cycle of the parent polymer P4' with the bifunctional crosslinker 1 . The folding is induced by UV-light ( $\lambda=320 \mathrm{~nm}$ ) resulting in the intramolecularly folded SCNPs P9 and P11. The unfolding of SCNP P9 was induced by mCPBA restoring the initial state (P10). 
Intriguingly, the PS-stat-CMS based polymer P4 (see Section A.7, $\dagger$ for structural details) displays luminescence (excitation wavelength $\lambda=240 \mathrm{~nm}$ ) in the range of about 270-375 $\mathrm{nm}$ (refer to the ESI Fig. S24a $\dagger$ ), while the luminescence is spin-silenced after the attachment of the nitroxide, yielding the profluorescent parent polymer $\mathbf{P 4}{ }^{\prime}$.

The use of light is a powerful tool to drive reactions, since light is readily available and readily controlled in intensity and wavelength. $\alpha$-Hydroxyalkyl phenyl ketones undergo a clean Norrish-type I cleavage upon irradiation to produce a benzoyl and the corresponding ketyl radical with high efficiency and quantum yields. ${ }^{52,53}$ Depending on the substituents in the para position of the aryl group, the cleavage of the $\mathrm{C}-\mathrm{C}$ bond can occur either from the singlet or the triplet excited state, and the photocleavage can involve either the $n-\pi^{*}$ or $\pi-\pi^{*}$ excited state. Thus, inspired by the photo-induced ligation of nitroxide radicals with the photoinitiator (2-hydroxy-1-(4-(2-hydroxyethoxy) phenyl)-2-methylpropan-1-one) (Irgacure 2959, an $\alpha$-hydroxyalkyl phenyl ketone derivative or a photoactive PS-based polymer), ${ }^{52,54}$ we targeted the intramolecular collapse induced by UV-light $\left(\lambda_{\max }=320 \mathrm{~nm}\right)$ of a nitroxide containing polymer through a novel bifunctional crosslinker 1 equipped with 2-hydroxy-4'-(2-hydroxyethoxy)-2-methylpropiophenone (Irgacure 2959) functionalities on both termini. 1 was prepared in a one-step synthesis by esterification of adipic acid with commercially available Irgacure 2959 (refer to the ESI for the synthesis (Section A.2†) and detailed characterization (Section C.1†)). Folding of $\mathbf{P 1} \mathbf{1}^{\prime}-\mathbf{P 4} \mathbf{4}^{\prime}$ was initially conducted by employing a stoichiometric amount of $\mathbf{1}$ (refer to ESI $\uparrow$ for the SEC traces of P1'-P4' and P5-P8, Fig. S17-S20 †). After irradiation by UV light, SEC of the polymers P5-P8 shows the characteristic shift towards higher retention times, due to the reduced hydrodynamic volume of the single-chain architectures (Fig. S17-S20 $\dagger$ ). In addition, the influence of the crosslinker equivalents on the SCNPs formation was investigated with regard to the pendant TEMPO groups, specifically for polymer P4' (Fig. 1a). Here, we observed a similar behaviour as the Berda team noted in their recent study. ${ }^{55}$ With increasing amount of crosslinker, a major peak shift towards higher retention times is detected. By employing 1 in large excess (25 eq.), interchain coupling of 1 is witnessed as a side reaction (refer to Fig. 1a, green curve). Thus, once the optimum folding conditions were established, 2 equivalents of $\mathbf{1}$ were used to affect a pronounced collapse visible in the SEC. As expected, the shift in the SEC chromatogram was detected (refer to Fig. 1b, red curve) resulting from the decrease of the hydrodynamic volume caused by the singlechain collapse. Importantly, the resulting SCNPs display inherent luminescence (excitation wavelength $\lambda=240 \mathrm{~nm}$ ) in the range of about $380-550 \mathrm{~nm}$, establishing a self-reporting reversible SCNPs folding system (compare the black and red lines in Fig. 1c). Critically, compared to other reported fluorescent SCNPs, ${ }^{56-59}$ it is not necessary to install a fluorophore before or after the crosslinking process, since the crosslinking reaction between the styryl TEMPO derivative and the $\alpha$-hydroxyalkyl phenyl ketones of the linker $\mathbf{1}$ generates the luminescence. The ${ }^{1} \mathrm{H}$ NMR spectrum of the folded SCNPs P9 displays the characteristic $\mathrm{CH}_{2}$ resonances of 1 at 4.21 and
$4.44 \mathrm{ppm}$ (Fig. S10 $\dagger$ ). In order to underpin the observation of luminescence switch-on (excitation wavelength $\lambda=240 \mathrm{~nm}$ ) after the crosslinking reaction, a monofunctional Irgacure 2959 derivative $\mathbf{2}$ was prepared and reacted with $\mathbf{P 4}$ ' to yield $\mathbf{P 9}$ ' (refer to Fig. 2). Interestingly, Jokusch et al. observed phosphorescence for the Irgacure 2959 moiety, ${ }^{60}$ giving a similar spectrum compared to the recorded luminescence spectra for the SCNPS P9 and P11. Furthermore, small molecule studies reveal no luminescence for the folded polymers P9 and P11. The luminescence is only detectable in case $\mathbf{1}$ or $\mathbf{2}$ are attached to the polymer (refer to Fig. 1c, $2 \mathrm{~b}$ and $\mathrm{S} 22 ; \dagger$ for further information for the small molecule study refer to the ESI Section A and $\mathrm{C} \dagger$ ). The peak at $480 \mathrm{~nm}$ is an artefact at double the wavelength that was used for excitation $(240 \mathrm{~nm})$, which was proven by the measurement of pure DCM (refer to Fig. S22 in the ESI $\dagger$ ). Additional confirmation of the folded nature of the product $\mathbf{P 9}$ was obtained by DOSY measurements. Applying the StokesEinstein equation (eqn (1) in the $\mathrm{ESI}_{\dagger} \dagger$ ), the hydrodynamic diameter ( $\left.D_{\mathrm{h}, \mathrm{DOSY}}\right)$ of the SCNPs of P9 is calculated to be $2.6 \mathrm{~nm}$ compared to $3.4 \mathrm{~nm}$ of the unfolded state of $\mathbf{P 4}^{\prime}$, which is in agreement with the DLS analysis (refer to Table 1, Fig. 1d, and Section C7-C8 in the ESI $\dagger$ for spectra and further details). The parent polymer and the respective SCNPs were further characterized by EPR in toluene to determine the radical structure as well as spin density of the samples (Fig. 2, black and red lines, respectively). The $g$ value and hyperfine coupling constants $\left(a_{\mathrm{N}}\right)$ for $\mathbf{P 4}^{\prime}$ (refer to Table S2 $\dagger$ ) agree with the classic TEMPO-based nitroxide structure. ${ }^{61}$ Thus, the EPR measurements of the folded sample P9 underpin the almost quantitative reaction of the nitroxide moieties with 1.

Based on the work of Chalmers et al., describing the deprotection of methoxy-protected TEMPO derivatives by metachloroperbenzoic acid ( $m$ CPBA) affording the hydroxyl amine which is then oxidized to the nitroxide radical, we reactivate the capped TEMPO species $\mathbf{P 4}^{\prime}$ by reoxidation yielding the hydroxylamine moiety, which subsequently reforms the nitroxide (refer to Scheme 1 and Fig. 1b, blue curve, and refer to Section A.11 in the $\mathrm{ESI} \dagger$ for the deprotection conditions).${ }^{62}$ Gratifyingly, the SEC chromatogram displays an almost identical molecular weight distribution for P10 compared to the original parent polymer P4'. Furthermore, the EPR data for P10 also suggest quantitative unfolding by restoring the initial amount of nitroxides in the polymeric backbone, confirming the results obtained by SEC (compare the values of the radical densities for TEMPO moiety shown in Table 1, and Fig. 3). In addition, the fluorescence spectrum of P10 depicts an identical shape as the unfolded precursor polymer $\mathbf{P 4}^{\prime}$ and no luminescence was monitored (Fig. 1c). Thus, the switch on/off behaviour of the luminescence is perfectly suited to assess the folded state of the SCNPs.

The dynamic-covalent folding/unfolding is similarly reflected in the DLS derived hydrodynamic diameter $\left(D_{\mathrm{h}}\right)$, which reverts back to larger hydrodynamic diameters (i.e., 3.2. $\mathrm{nm}$ ), indicating the successful unfolding (refer to Fig. 1d, blue curve and Table 1 ). In addition, the $D_{\mathrm{h}, \mathrm{DOSY}}$ derived value of the SCNP for P10 is close to $3.5 \mathrm{~nm}$, which is in agreement with the DLS analysis (Table 1 and Fig. S36-S37†). The ${ }^{1} \mathrm{H}$ NMR spectrum of 
a)

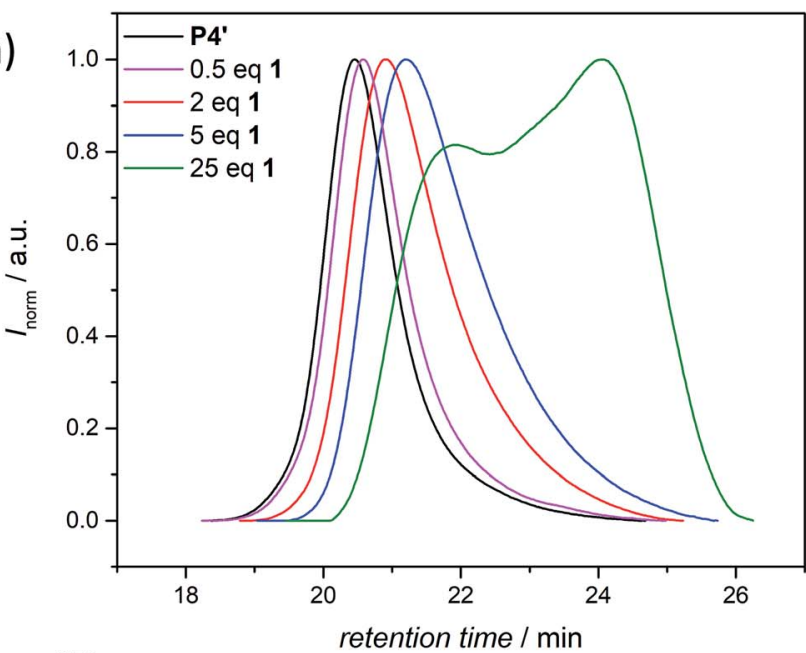

c)

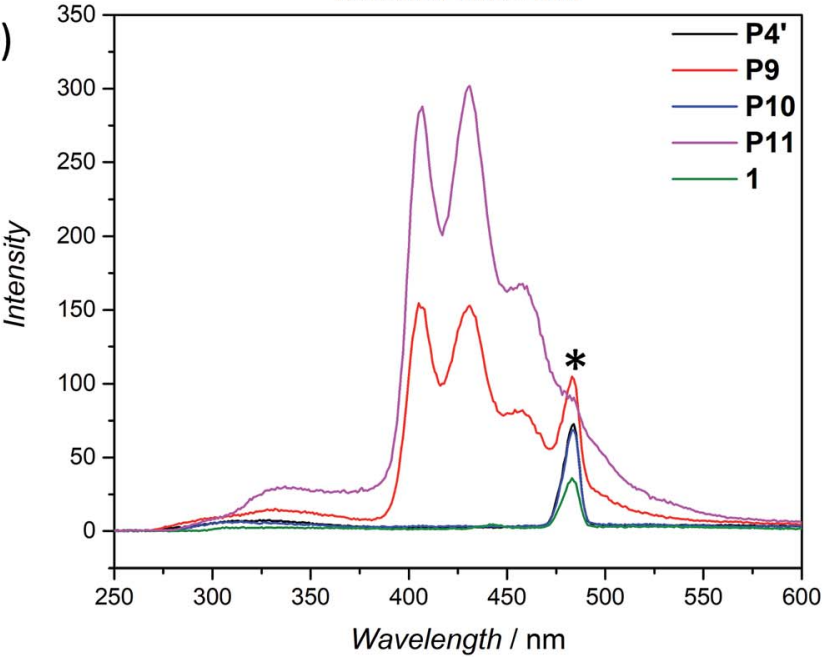

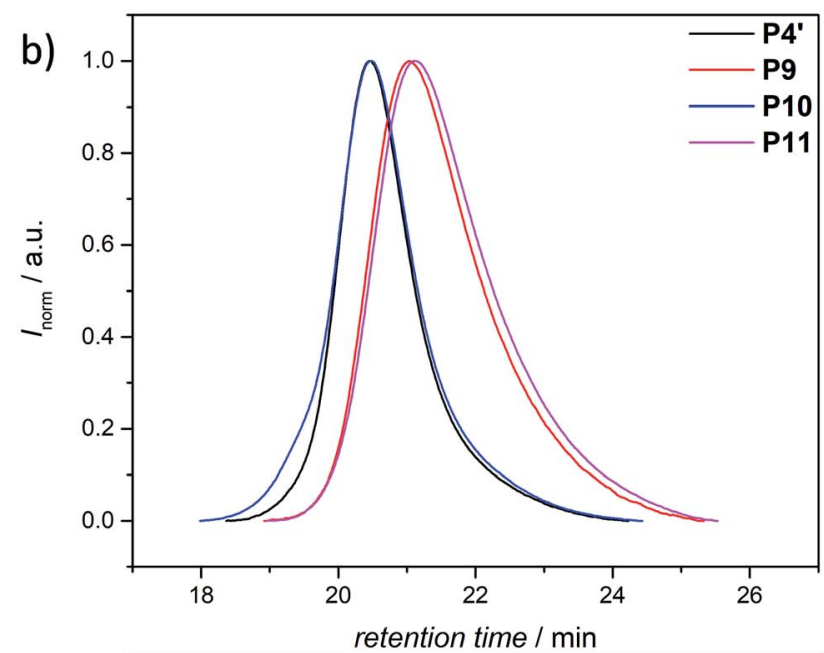

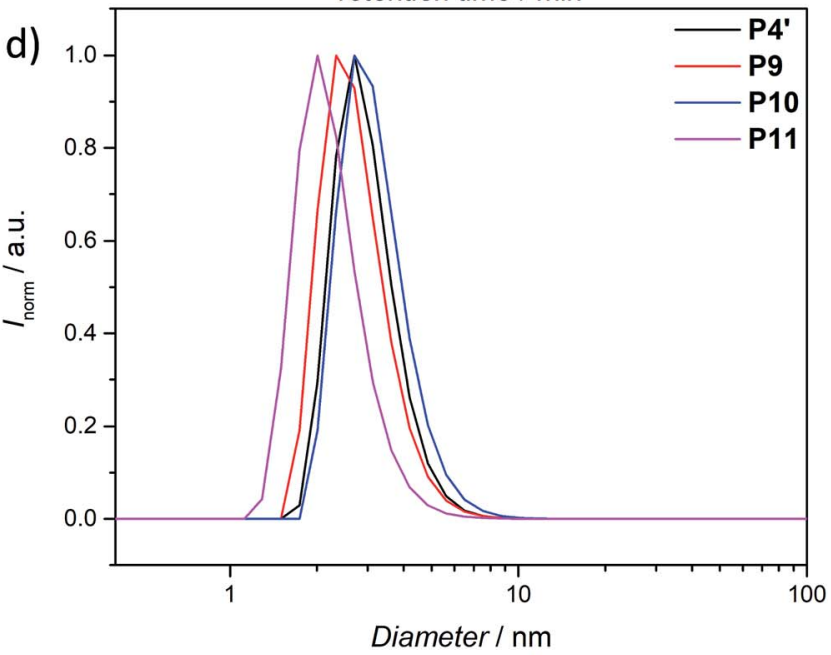

Fig. 1 (a) SEC chromatogram of P4' (black curve) and the SEC curves of the folded polymer P9 as a function of the amount of added crosslinker 1. (b) Folding cycle of the TEMPO polymer P4'. The black curve shows the initial precursor polymer, red curve shows the folded polymer, the blue curve shows the unfolded SCNP followed by the refolding (pink curve). (c) Emission spectra ( $\lambda_{\text {exc. }}=240 \mathrm{~nm}$ ) of the polymers P4', P9-P11 as well as the crosslinker 1 , measured in DCM $\left(c=0.2 \mathrm{mg} \mathrm{mL}^{-1}\right)$. The peak marked with a star is a systematic artefact at double the excited wavelength. (d) Analysis of P4', P9-P11 via dynamic light scattering (DLS) in DMAc + 0.3\% LiBr, the average (minimum of 3 measurements per sample) number distributions are shown.

the unfolded SCNPs P10 (refer to Fig. S9†) shows a decrease of the characteristic $\mathrm{CH}_{2}$ resonances of 1 at 4.21 and $4.44 \mathrm{ppm}$ by close to $82 \%$. These results are congruent with the reported yields for the deprotection of nitroxide moieties by Chalmers et $a .^{62}$ Hence, not all nitroxide radicals are completely recovered, although the unfolding appears to be complete. We assume that only one functionality of the bifunctional crosslinker is cleaved, while the second functionality is still attached to the polymer. The latter assumption is confirmed by above discussed SEC and DLS results, where slightly higher apparent molecular weight and diameter values were recorded for $\mathbf{P 1 0}$ compared to $\mathbf{P 4}^{\prime}$. In addition, the slight decrease in the signal intensity of $\mathbf{P 1 0}$ in comparison to $\mathbf{P 4} \mathbf{4}^{\prime}$ observed during the EPR measurements (refer Fig. 3, blue and black lines, respectively) indicate a slightly lower spin density, thus reaffirming the aforementioned assumption of the cleavage of one crosslink site, while the second crosslink is still attached to the nitroxide.
After the SCNPs P9 has been successfully unfolded, we implemented the UV-light induced refolding reaction of 1 with P10 to unambiguously prove the reformation of the nitroxide radicals (refer to Scheme 1). In a similar manner to the initial SCNPs formation studies, 2 equivalents of 1 were used in order to effect a pronounced collapse visible in the SEC. Indeed, the expected shift in the SEC chromatogram was detected (refer to Fig. 1b, pink curve), resulting from the decrease of the hydrodynamic volume caused by the single-chain collapse. Surprisingly, the shift of the refolded SCNP P11 is more pronounced as the initial SCNPs P9. In fact, $m$ CPBA is often used as oxidizing agent for the generation of nitroxides from piperidine derivatives $^{46}$ as well as deprotection agent as reported by Chalmers et al. Consequently, the unfolding reaction may regenerate some deactivated nitroxide radicals that were not accessible for the first folding reaction as well as restore the crosslinked moieties. The SEC data underpinning the second folding cycle 
a)

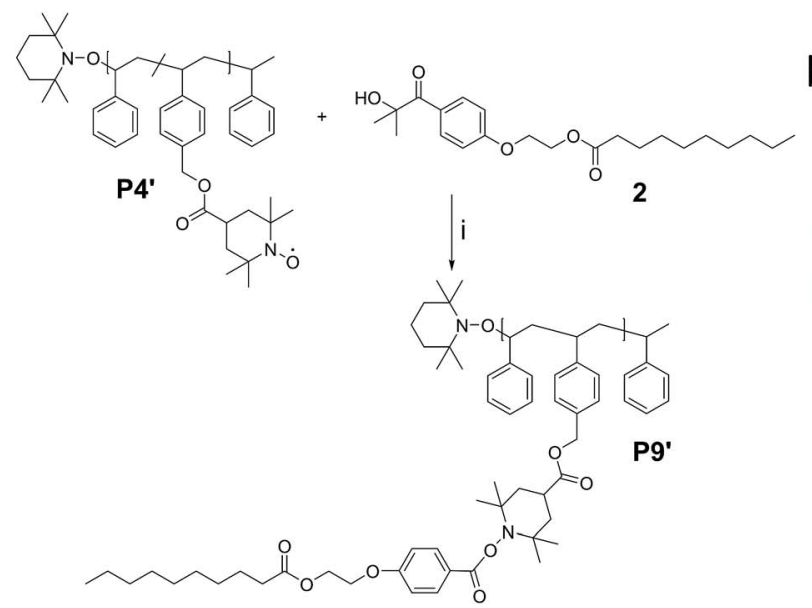

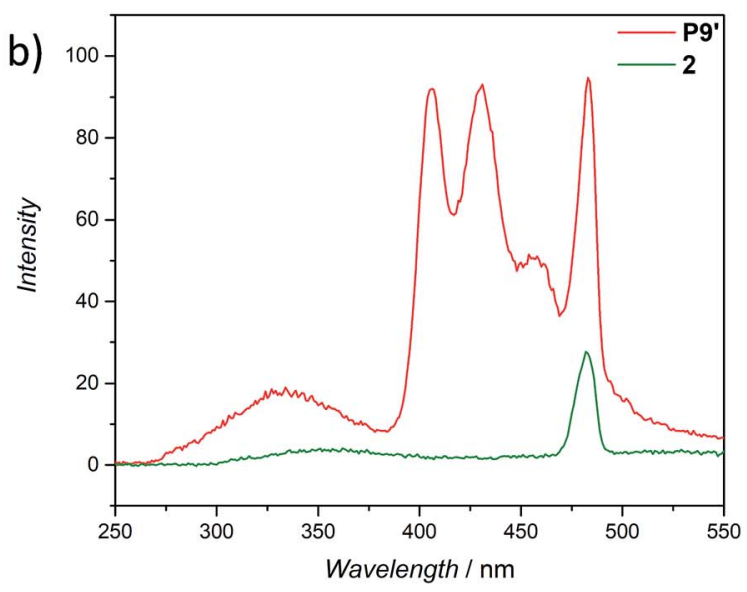

Fig. 2 Synthesis of a non-collapsed analogue to $\mathrm{P9}\left(\mathrm{Pg}^{\prime}\right)$ via the UV-light induced reaction of the precursor polymer $\mathrm{P} 4^{\prime}$ and the photo-active monofunctional crosslinker 2. (a) (i) $h \nu$ ( $\lambda_{\max }=320 \mathrm{~nm}$ ), ambient temperature, $20 \mathrm{~h}$. (b) Fluorescence spectra of the mono-functional crosslinker 2 and $\mathrm{P9}^{\prime}$, demonstrating the fluorescent nature of the molecular construct.

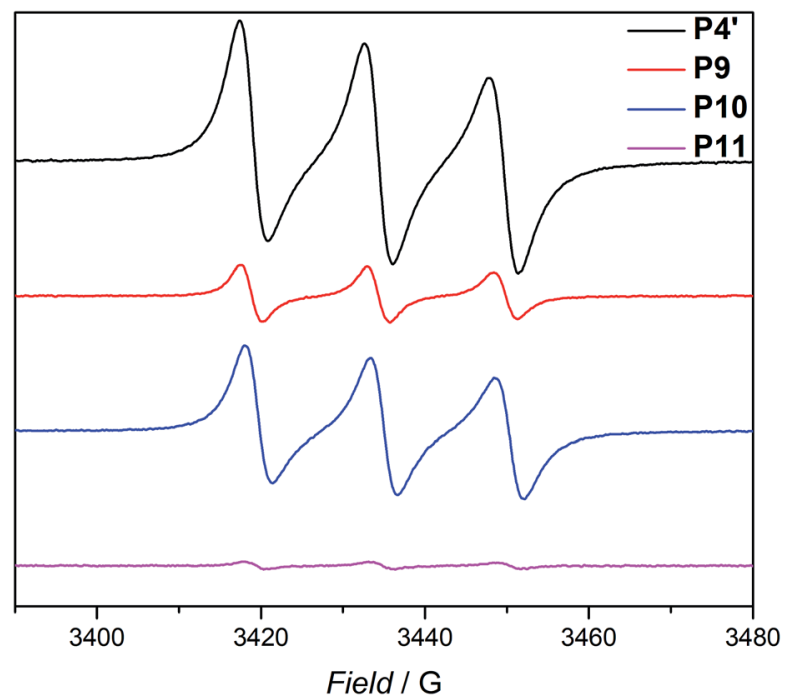

Fig. 3 EPR signals for the parent polymer P4', the folded SCNP P9, the unfolded P10 and refolded SCNP P11. All samples were measured in toluene at ambient temperature.

are corroborated by fluorescence and DLS data. The fluorescence spectrum (refer to Fig. 1c) shows higher intensity (pink curve) compared to the initial folded state (red curve), which indicates more crosslinking points are formed, allowing for a more distinct luminescence. The fluorescence quantum yield after the second folding is increased by close to a factor of 150 (refer to Fig. S27 in the ESI $\dagger$ ). Importantly, the DLS derived $D_{\mathrm{h}}$ values are also congruent with the above results: the initially folded SCNPs (Fig. 1d, red curve) are less compact than the refolded system (Fig. 1d, pink curve), i.e. $D_{\mathrm{h}}$ going from $2.8 \mathrm{~nm}$ to $2.3 \mathrm{~nm}$ after the first and second folding, respectively. In addition, the size reduction after the second folding was monitored by DOSY measurements (refer Table 1, Fig. S38S39†). Similar to DLS and SEC, DOSY provides information about the hydrodynamic radii by measuring the diffusion coefficient, which should typically increase upon folding, thus resulting in a smaller $D_{\mathrm{h}}$ value. Based on the Stokes-Einstein equation, the calculated $D_{\mathrm{h}, \mathrm{DOsy}}$ shows the same trend, thus a decreasing $D_{\mathrm{h}}$ from 3.5 to $2.9 \mathrm{~nm}$ for the refolded P11 as beforehand accessed by DLS. Finally, by the virtue of the EPR experiments, a very significant decrease in the signal intensity (Fig. 2, pink line) upon the SCNPs folding process is evident, which implies the superior folding of polymer P10 to SCNPs P11.

\section{Conclusions}

In summary, we pioneer a refoldable, self-reporting singlechain nanoparticle platform technology based on the photoligation of nitroxide radicals with a novel bifunctional crosslinker bearing a photoreactive functionality. The initially folded, luminescent SCNPs are unfolded under oxidative conditions, resulting in luminescence switch-off and the reestablishment of the initial precursor polymer. Subsequently, the unfolded polymer was refolded by the same photo-induced reaction, resulting in the restoration of the luminescence (refer to Scheme 1 and Fig. 3) and the characteristic molecular and hydrodynamic diameter changes. We submit that the introduced photochemical reversible SCNP folding system is the first to indicate its folded or unfolded state by a simple luminescence readout. The technology presented herein may thus serve as a guiding principle to readout the state of SCNPs in more complex scenarios. Furthermore, the presented principle could be adopted in other fields to distinguish between small molecular (non-luminescent) species and polymeric luminescent structures.

\section{Conflicts of interest}

There are no conflicts to declare. 


\section{Acknowledgements}

C. B.-K. and B. L. acknowledges funding for this project from the Sonderforschungsbereich 1176 (project A1) funded by the German Research Council (DFG). Additional long term support from the Karlsruhe Institute of Technology (KIT), the Helmholtz association via the BioInterfaces in Technology and Medicine (BIFTM) and the Science and Technology of Nanosystems (STN) programs is acknowledged. C. B.-K. acknowledges continued support from the Queensland University of Technology (QUT) and the Australian Research Council (ARC) in the form of a Laureate fellowship enabling his photochemical research program. The authors acknowledge Prof. M. Meier and Dr P. Levkin (both KIT) respectively for the access to ATR-IR and DLS equipment.

\section{References}

1 O. Altintas and C. Barner-Kowollik, Macromol. Rapid Commun., 2012, 33, 958-971.

2 C. K. Lyon, A. Prasher, A. M. Hanlon, B. T. Tuten, C. A. Tooley, P. G. Frank and E. B. Berda, Polym. Chem., 2015, 6, 181-197.

3 M. Ouchi, N. Badi, J.-F. Lutz and M. Sawamoto, Nat. Chem., 2011, 3, 917-924.

4 A. M. Hanlon, C. K. Lyon and E. B. Berda, Macromolecules, 2016, 49, 2-14.

5 M. Gonzalez-Burgos, A. Latorre-Sanchez and J. A. Pomposo, Chem. Soc. Rev., 2015, 44, 6122-6142.

6 R. K. Roy and J.-F. Lutz, J. Am. Chem. Soc., 2014, 136, 1288812891.

7 S. Mavila, O. Eivgi, I. Berkovich and N. G. Lemcoff, Chem. Rev., 2016, 116, 878-961.

8 O. Altintas and C. Barner-Kowollik, Macromol. Rapid Commun., 2016, 37, 29-46.

9 T. S. Fischer, D. Schulze-Sünninghausen, B. Luy, O. Altintas and C. Barner-Kowollik, Angew. Chem., Int. Ed., 2016, 55, 11276-11280.

10 O. Altintas, M. Artar, G. Ter Huurne, I. K. Voets, A. R. A. Palmans, C. Barner-Kowollik and E. W. Meijer, Macromolecules, 2015, 48, 8921-8932.

11 E. A. Appel, J. Dyson, J. Delbarrio, Z. Walsh and O. A. Scherman, Angew. Chem., Int. Ed., 2012, 51, 4185-4189.

12 N. Hosono, M. A. J. Gillissen, Y. Li, S. S. Sheiko, A. R. A. Palmans and E. W. Meijer, J. Am. Chem. Soc., 2013, 135, 501-510.

13 F. Wang, H. Pu and X. Che, Chem. Commun., 2016, 52, 35163519.

14 S. Mavila, I. Rozenberg and N. G. Lemcoff, Chem. Sci., 2014, 5, 4196-4203.

15 A. Sanchez-Sanchez, A. Arbe, J. Colmenero and J. A. Pomposo, ACS Macro Lett., 2014, 3, 439-443.

16 B. T. Tuten, D. Chao, K. Lyon and E. B. Berda, Polym. Chem., 2012, 3, 3068-3071.

17 N. Wedler-Jasinski, T. Lueckerath, H. Mutlu, A. S. Goldmann, A. Walther, M. H. Stenzel and C. BarnerKowollik, Chem. Commun., 2017, 6-9.
18 B. S. Murray and D. A. Fulton, Macromolecules, 2011, 44, 7242-7252.

19 J. Zhang, J. Tanaka, P. Gurnani, P. Wilson, M. Hartlieb and S. Perrier, Polym. Chem., 2017, 8, 4079-4089.

20 A. Sanchez-Sanchez, D. A. Fulton and J. A. Pomposo, Chem. Commun., 2014, 50, 1871-1874.

21 R. Lambert, A.-L. Wirotius and D. Taton, ACS Macro Lett., 2017, 6, 489-494.

22 J. Zhang, G. Gody, M. Hartlieb, S. Catrouillet, J. Moffat and S. Perrier, Macromolecules, 2016, 49, 8933-8942.

23 K. S. Park, D. Y. Kim, S. K. Choi and D. H. Suh, Jpn. J. Appl. Phys., 2003, 42, 3877-3880.

24 J. Jiang and S. Thayumanavan, Macromolecules, 2005, 38, 5886-5891.

25 D. Mecerreyes, V. Lee, C. J. Hawker, J. L. Hedrick, A. Wursch, W. Volksen, T. Magbitang, E. Huang and R. D. Miller, Adv. Mater., 2001, 13, 204-208.

26 A. M. Hanlon, R. Chen, K. J. Rodriguez, C. Willis, J. G. Dickinson, M. Cashman and E. B. Berda, Macromolecules, 2017, 50, 2996-3003.

27 J. Willenbacher, K. N. R. Wuest, J. O. Mueller, M. Kaupp, H.-A. Wagenknecht and C. Barner-Kowollik, ACS Macro Lett., 2014, 3, 574-579.

28 T. K. Claus, J. Zhang, L. Martin, M. Hartlieb, H. Mutlu, S. Perrier, G. Delaittre and C. Barner-Kowollik, Macromol. Rapid Commun., 2017, 38, 1700264.

29 C. Heiler, J. T. Offenloch, E. Blasco and C. Barner-Kowollik, ACS Macro Lett., 2016, 6, 56-61.

30 O. Altintas, J. Willenbacher, K. N. R. Wuest, K. K. Oehlenschlaeger, P. Krolla-Sidenstein, H. Gliemann and C. Barner-Kowollik, Macromolecules, 2013, 46, 8092-8101.

31 P. G. Frank, B. T. Tuten, A. Prasher, D. Chao and E. B. Berda, Macromol. Rapid Commun., 2014, 35, 249-253.

32 W. Fan, X. Tong, Q. Yan, S. Fu and Y. Zhao, Chem. Commun., 2014, 50, 13492-13494.

33 I. Perez-Baena, I. Asenjo-Sanz, A. Arbe, A. J. Moreno, F. Lo Verso, J. Colmenero and J. A. Pomposo, Macromolecules, 2014, 47, 8270-8280.

34 J. Winsberg, T. Janoschka, S. Morgenstern, T. Hagemann, S. Muench, G. Hauffman, J. F. Gohy, M. D. Hager and U. S. Schubert, Adv. Mater., 2016, 28, 2238-2243.

35 J. Winsberg, S. Muench, T. Hagemann, S. Morgenstern, T. Janoschka, M. Billing, F. H. Schacher, G. Hauffman, J.-F. Gohy, S. Hoeppener, M. D. Hager and U. S. Schubert, Polym. Chem., 2016, 7, 1711-1718.

36 T. Janoschka, N. Martin, U. Martin, C. Friebe, S. Morgenstern, H. Hiller, M. D. Hager and U. S. Schubert, Nature, 2015, 527, 78-81.

37 S. E. Allen, R. R. Walvoord, R. Padilla-Salinas and M. C. Kozlowski, Chem. Rev., 2013, 113, 6234-6458.

38 C. L. Rayner, S. E. Bottle, G. A. Gole, M. S. Ward and N. L. Barnett, Neurochem. Int., 2016, 92, 1-12.

39 T. S. Fischer, J. Steinkoenig, H. Woehlk, J. P. Blinco, K. Fairfull-Smith and C. Barner-Kowollik, Polym. Chem., 2017, 8, 5269-5274.

40 L. Tebben and A. Studer, Angew. Chem., Int. Ed., 2011, 50, 5034-5068. 
41 T. Janoschka, A. Teichler, A. Krieg, M. D. Hager and U. S. Schubert, J. Polym. Sci., Part A: Polym. Chem., 2012, 50, 1394-1407.

42 R. K. Roy, A. Meszynska, C. Laure, L. Charles, C. Verchin and J.-F. Lutz, Nat. Commun., 2015, 6, 7237-7239.

43 K. Zhang, M. J. Monteiro and Z. Jia, Polym. Chem., 2016, 7, 5589-5614.

44 J. P. Blinco, D. J. Keddie, T. Wade, P. J. Barker, G. A. George and S. E. Bottle, Polym. Degrad. Stab., 2008, 93, 1613-1618.

45 V. Vitzthum, F. Borcard, S. Jannin, M. Morin, P. Miéville, M. A. Caporini, A. Sienkiewicz, S. Gerber-Lemaire and G. Bodenhausen, ChemPhysChem, 2011, 12, 2929-2932.

46 H. Mutlu, C. W. Schmitt, N. Jasinski, H. Woehlk, K. FairfullSmith, J. P. Blinco and C. Barner-Kowollik, Polym. Chem., 2017, 8, 6199-6203.

47 E. M. Simpson, Z. D. Ristovski, S. E. Bottle, K. E. FairfullSmith and J. P. Blinco, Polym. Chem., 2015, 6, 2962-2969.

48 K. Hansen, K. E. Fairfull-Smith, S. E. Bottle and J. P. Blinco, Macromol. Chem. Phys., 2016, 217, 2330-2340.

49 A. Kuo, Chem, 2011, 1, 80-86.

50 A. Kuo, Chem, 2011, 1, 40-51.

51 J. Willenbacher, O. Altintas, V. Trouillet, N. Knöfel, M. J. Monteiro, P. W. Roesky and C. Barner-Kowollik, Polym. Chem., 2015, 6, 4358-4365.

52 A. Mardyukov and A. Studer, Macromol. Rapid Commun., 2013, 34, 94-101.
53 F. Mäsing, A. Mardyukov, C. Doerenkamp, H. Eckert, U. Malkus, H. Nüsse, J. Klingauf and A. Studer, Angew. Chem., Int. Ed., 2015, 54, 12612-12617.

54 G. Delaittre, M. Dietrich, J. P. Blinco, A. Hirschbiel, M. Bruns, L. Barner and C. Barner-Kowollik, Biomacromolecules, 2012, 13, 1700-1705.

55 A. M. Hanlon, I. Martin, E. R. Bright, J. Chouinard, K. J. Rodriguez, G. E. Patenotte and E. B. Berda, Polym. Chem., 2017, 8, 5120-5128.

56 Y. Bai, H. Xing, G. A. Vincil, J. Lee, E. J. Henderson, Y. Lu, N. G. Lemcoff and S. C. Zimmerman, Chem. Sci., 2014, 5, 2862-2868.

57 S. K. Hamilton and E. Harth, ACS Nano, 2009, 3, 402-410.

58 P. Wang, H. Pu, J. Ge, M. Jin, H. Pan, Z. Chang and D. Wan, Mater. Lett., 2014, 132, 102-105.

59 J. De-La-Cuesta, E. Gonzalez and J. A. Pomposo, Molecules, 2017, 22, 1819.

60 S. Jockusch, M. S. Landis, B. Freiermuth and N. J. Turro, Macromolecules, 2001, 34, 1619-1626.

61 W. Snipes, J. Cupp, G. Cohn and A. Keith, Biophys. J., 1974, 14, 20-32.

62 B. A. Chalmers, J. C. Morris, K. E. Fairfull-Smith, R. S. Grainger and S. E. Bottle, Chem. Commun., 2013, 49, 10382-10384. 